\title{
Bioengineering, a Cadmium Sensing Green Fluorescent Protein, Based Whole-Cell Biosensor From Pseudomonas
}

\section{aeruginosa}

\author{
Krishani Dave ${ }^{1}$ (D) \\ 1 Department of Applied Sciences, Northumbria University, City Campus, Sutherland Building, Newcastle- Upon Tyne, \\ NE1 8ST United Kingdom; krishani.dave@gmail.com (K.D.); \\ * Correspondence: krishani.dave@gmail.com;
}

Received: 5.09.2020; Revised: 2.10.2020; Accepted: 4.10.2020; Published: 6.10.2020

\begin{abstract}
Heavy metal toxicity is a significant issue due to an increase in industrial waste production, with cadmium being one of the major pollutants. An approach involving homologous cloning was made to bioengineer a microbial cadmium biosensor from a strain of Pseudomonas aeruginosa. The promoter pCadR from $P$. aeruginosa was cloned into a vector pEGFP-N2 using Gibson assembly. Escherichia coli DH5 alpha strain was used as the host cell, which on sensing cadmium, produced fluorescence using the reporter gene called green fluorescent protein (GFP). The clone, pEGFP-N2 $2_{\text {CadR, }}$ was subjected to increasing concentrations of cadmium chloride to determine the sensitivity. It was observed that pEGFP-N2 $2_{\text {CadR }}$ responded to micromolar concentrations of cadmium chloride; however, it was determined that the biosensor tested it with lead nitrate and copper sulfate solutions had non-specific interactions with other metals. The interaction of the promoter with the metals was weak compared to previous studies, which was attributed to several reasons mentioned in the paper. As the sensor's fluorescent intensities were dull and indistinguishable, it was not classified as a useful cadmium biosensor. Further studies for determining the promoter interaction and affinity towards various metals at varying concentrations are required to validate the results obtained.
\end{abstract}

Keywords: whole-cell biosensor; Pseudomonas aeruginosa; cadmium; green fluorescent protein; heavy metal toxicity.

(C) 2020 by the authors. This article is an open-access article distributed under the terms and conditions of the Creative Commons Attribution (CC BY) license (https://creativecommons.org/licenses/by/4.0/).

\section{Introduction}

Metals are an essential part of the ecosystem. The living organisms utilize various metals like iron, magnesium, etc., for crucial processes that involve growth and cellular metabolism. However, there are certain metals classified as heavy metals that are considered toxic to the environment. They can be defined as a metal group with an atomic density greater than $5 \mathrm{~g} / \mathrm{cm}^{3}$ [1]. Particular examples of heavy metals include lead (Pb), Zinc ( $\left.\mathrm{Zn}\right)$, and Cadmium $(\mathrm{Cd})$. Heavy metal content in the terrestrial and aquatic ecosystem has increased due to industrial waste's inappropriate disposal and increased urbanization and industrialization. Due to their bioaccumulative nature, they pose a significant threat to the food chain [2]. The toxicity of heavy metals comes from producing high amounts of reactive oxygen species [3].

Out of all the metals, cadmium is one of the major contaminants causing serious problems. It is extremely poisonous [4]. Some of the important incidences involving cadmium poisoning are Itai Itai disease of Japan and high cadmium concentration in the kidneys of willow ptarmigan birds in the mine-belt region of the United States [5,6]. At low https://nanobioletters.com/ 
concentrations, cadmium can bind to the mitochondria inhibiting oxidative phosphorylation and cellular respiration. It can cause chromosomal aberrations, DNA strand break, and exchange of sister chromatids. Cadmium toxicity can also deplete glutathione, which is an antioxidant, increasing ROS formation furthermore. It also possesses the capability to inhibit antioxidative enzymes like superoxide dismutase and catalase [6].

These issues make it necessary to detect the concentration of cadmium in the environment to facilitate the remedial process. Some of the conventional methods used for the detection include laser-induced breakdown spectroscopy [7], atomic absorption spectroscopy, inductively coupled plasma mass spectroscopy [8]. However, these conventional methods have disadvantages like - being time-consuming, non-cost effective, and often require extensive sample preparation, which can only be done by trained personnel [9].

An alternative to these, recent focus has switched to developing whole-cell heavy metal biosensors for detecting a certain amount of a particular heavy metal, like cadmium. Certain bacteria, when subjected continuously to cadmium, develop resistance against it. The metal resistance operon system developed by these bacteria can make the biosensor as they can survive under defined concentrations of this metal [9]. This method is widely recognized due to its ease of production, simple measurement techniques, and cost-effectiveness. A typical description of a whole-cell biosensor is a sensor protein targeting a ligand of interest, which, when coupled with a genetic regulatory system, produces a measurable signal in the host cell [10]. This host cell is generally a bacterium genetically engineered to respond to cadmium levels through a reporter gene [11].

The regulatory proteins that respond to the metal are classified into two major types: MerR and ArsR family. MerR family confers resistance to many other metals such as cadmium, copper, lead, zinc, and mercury. The cadmium regulatory protein called CadR belongs to the MerR family and confers resistance to cadmium. In this study, the promoter of CadR, was used to bind to cadmium with high affinity in certain species of pseudomonas, especially Pseudomonas aeruginosa, allowing these bacteria to survive in high concentrations of cadmium [8].

Most of the experiments use traditional cloning techniques to make the whole-cell bacterial biosensors. In this experiment, a newer approach in creating the biosensor was implied. The Gibson assembly cloning technique was used to make the final validation clone. This method offers several advantages over traditional cloning methods 1) it is faster than traditional cloning and can be completed within 60 minutes, 2) it can join any two fragments without relying on compatible restriction sites, 3) it is seamlessly efficient, allowing multiple fragment directional cloning avoiding the need for multiple rounds of cloning, 4) it can clone fragments from up to $100 \mathrm{bp}$ to $100 \mathrm{~kb}$ with high cloning efficiencies 5) it minimizes errors resulting in a high percentage of error-free constructs due to its proofreading polymerase included in the kit [12].

This experiment aimed to develop a qualitative cadmium sensing biosensor using green fluorescent protein as the reporter gene. This experiment hypothesizes that the biosensor's fluorescence intensity will significantly increase with rising concentrations of the heavy metal cadmium. There will also be a significant difference between the intensities of different metals. 


\section{Materials and Methods}

\subsection{Determining the promoter region.}

The promoter region called pCadR was determined using the website called ensemble bacteria. The promoter sequence mentioned for $\mathrm{pCadR}$ by Prabhakaran and his colleagues was used to blast the genome of $P$. aeruginosa [8]. After the addition of flanking regions, the complete sequence with primer sites was determined. The $P$. aeruginosa strain cultured from glycerol stock was unclassified. To discover $P$. aeruginosa's strain in this study, the sequence obtained was blasted against $P$. aeruginosa database. For downstream analysis, PA7 was chosen as it appeared to be the most well characterized. Another reason for choosing this strain was that Prabhakaran's research had mentioned that the BLAST of CadR showed the highest homology (94.9\%) with the $\mathrm{Cd}(\mathrm{II}) / \mathrm{Pb}$ (II) - a responsive transcriptional regulator of PA7 [8]. This signified that this strain's promoter region could also be a good promoter for cadmium and could be used for this study.

\subsection{Molecular cloning of pCadR into E. coli DH5 $\alpha$.}

\subsubsection{Culture conditions for $P$. aeruginosa.}

$5 \mathrm{~mL}$ Luria Bertani broth was prepared. The broth was then inoculated with a glycerol stock containing $P$. aeruginosa. The bacteria were grown overnight at $37^{\circ} \mathrm{C}$ in an orbital shaker at 200 RPM.

\subsubsection{Genomic DNA extraction of P. aeruginosa.}

One $\mathrm{mL}$ of the overnight culture was centrifuged at $14000 \mathrm{RPM}$ for 2 minutes to pellet the cells. Nuclei lysis solution was added, and the tube was incubated at $80^{\circ} \mathrm{C}$ for 5 minutes to lyse the cells. RNase Solution was added to the cell lysate. After 60 minutes of incubation at $37^{\circ} \mathrm{C}$, the sample was cooled to room temperature(RT). Protein Precipitation Solution was added, and the tube was vortexed. The sample was incubated on ice for 5 minutes and then centrifuged. The supernatant was transferred to a clean microcentrifuge tube containing RT isopropanol and was mixed by inversion. The tube was centrifuged, and the supernatant was poured off. DNA pellet was washed by adding RT 70\% ethanol. The alcohol was aspirated after re-centrifugation. The pellet was allowed to air-dry for 10-15 minutes and was rehydrated with DNA Rehydration Solution. The tube was incubated at $65^{\circ} \mathrm{C}$ for 1 hour. Following incubation, the DNA was stored at $4^{\circ} \mathrm{C}$. The epoch microplate spectrophotometer from BioTek instruments was used to measure the DNA concentration. Primer sequences are mentioned in table 2 .

\subsubsection{PCR reaction for genomic DNA.}

\subsubsection{Primer reconstitution.}

The primers were ordered from Biobasic Canada incorporation. The primers were diluted according to the volumes provided by the manufacturer's instruction manual - forward primer with $221 \mu \mathrm{L}$ and reverse primer with $267 \mu \mathrm{L}$ of sterile water. 


\subsubsection{PCR reaction.}

The tube was subjected to PCR and gel electrophoresis. PCR machine used was the BIOER life ECO thermocycler. The ladder size used for gel electrophoresis was $1 \mathrm{~KB}$ plus (from New England Biolabs). The sample and ladder were loaded onto the gel (1.2\% agarose), and the electrophoresis was run at $100 \mathrm{~V}$ for 60 minutes.

\subsubsection{PCR product purification.}

The product was purified using the GeneJET PCR purification kit from ThermoFisher Scientific. Binding buffer was added to the completed PCR mixture, which was followed by the addition of $100 \%$ isopropanol. The solution was transferred to geneJET purification column and was centrifuged at 14000 RPM for 2 minutes. The flow-through was discarded, and this step was repeated until the whole solution had been added to the tube membrane. Wash buffer was added to the column, and the flow-through was discarded following centrifugation. The purification column was transferred to a clean $1.5 \mathrm{~mL}$ microcentrifuge tube to which elution buffer was added. The tube was centrifuged, and the purification column was discarded. The DNA was stored at $-20^{\circ} \mathrm{C}$.

\subsubsection{Amplification of pEGFP-N2 vector.}

The vector used for Gibson assembly was pEGFPN2, as it already contained the EGFP gene in the plasmid. The vector was amplified using the Q5 DNA polymerase protocol with the PCR conditions mentioned in table 1. Primer sequences are mentioned in table 2.

Table 1. Conditions to run PCR for amplification of vector pEGFP-N2.

\begin{tabular}{c|c|c|c} 
STEP & TEMP $^{\mathbf{0}} \mathbf{C}$ & TIME & NO. OF CYCLES \\
\hline Initial denaturation & 98 & $30 \mathrm{~s}$ & 1 \\
\hline Denaturation & 98 & $10 \mathrm{~s}$ & 35 \\
\hline Annealing & 58 & $30 \mathrm{~s}$ & 35 \\
\hline Extension & 72 & $3 \mathrm{~m}$ & 35 \\
\hline Final Extension & 72 & $10 \mathrm{~m}$ & 1 \\
\hline Hold & 4 & Indefinite & Indefinite
\end{tabular}

2.1.5. Cloning promoter into pEGFPN2 vector using Gibson assembly.

\subsubsection{Amplification of fragment to be inserted (promoter).}

The primers were reconstituted and diluted to $5 \mu \mathrm{M}$. The reagents were added to the tube following the manufacture's protocol for amplifying the fragments to create overhanging regions for Gibson assembly. The fragments were subjected to PCR and Gel electrophoresis (100 V for 60 minutes).

\subsubsection{Gibson assembly reaction.}

The concentration of products needed was calculated using the following formula to dilute accordingly. 
Pmol $=\underline{\text { (weight in ng) } \times 1000}$

Base pairs x 650 Daltons

As the insert size in this study was $177 \mathrm{bp}$, which was less than 200bp, the vector concentration was multiplied by 5 to optimize the cloning efficiency. Based on the concentration of pmol calculated, the amount of insert was calculated in ng.

The thermocycler was set up at $50^{\circ} \mathrm{C}$, and Gibson assembly was run at $50^{\circ} \mathrm{C}$ for 1 hour. The vector clone was called pEGFP-N2 CadR. The mutant vector was transformed into NEB 5alpha competent E.coli ordered from New England Biolabs, on a kanamycin plate (the vector possesses kanamycin resistance). The final concentration of kanamycin used was $50 \mu \mathrm{g} / \mathrm{mL}$. A colony PCR reaction was run, and gel electrophoresis was performed to determine the size of the pEGFP-N2Cadr. The positive clone with a 4150 bp band was subjected to plasmid extraction and was subsequently sent for sequencing. Primer sequences are mentioned in table 2.

Table 2. A tabulated summary of the primer name and sequences used for this study.

\begin{tabular}{c|c|c} 
PRIMER NAME & PRIMER SEQUENCE (5'-3') & REACTION \\
\hline pCadRF & GCTCGTAGTAGCGGATGGTC & Genomic DNA \\
PCR reaction
\end{tabular}

Ape software was used to construct a visual representation of the complete Gibson assembly product, as observed in figure 1. The promoter fragment in yellow drives the expression of the eGFP, which is a part of the vector pEGFP-N2. From this diagram, it was concluded that the final product should have a size of $4150 \mathrm{bp}$.

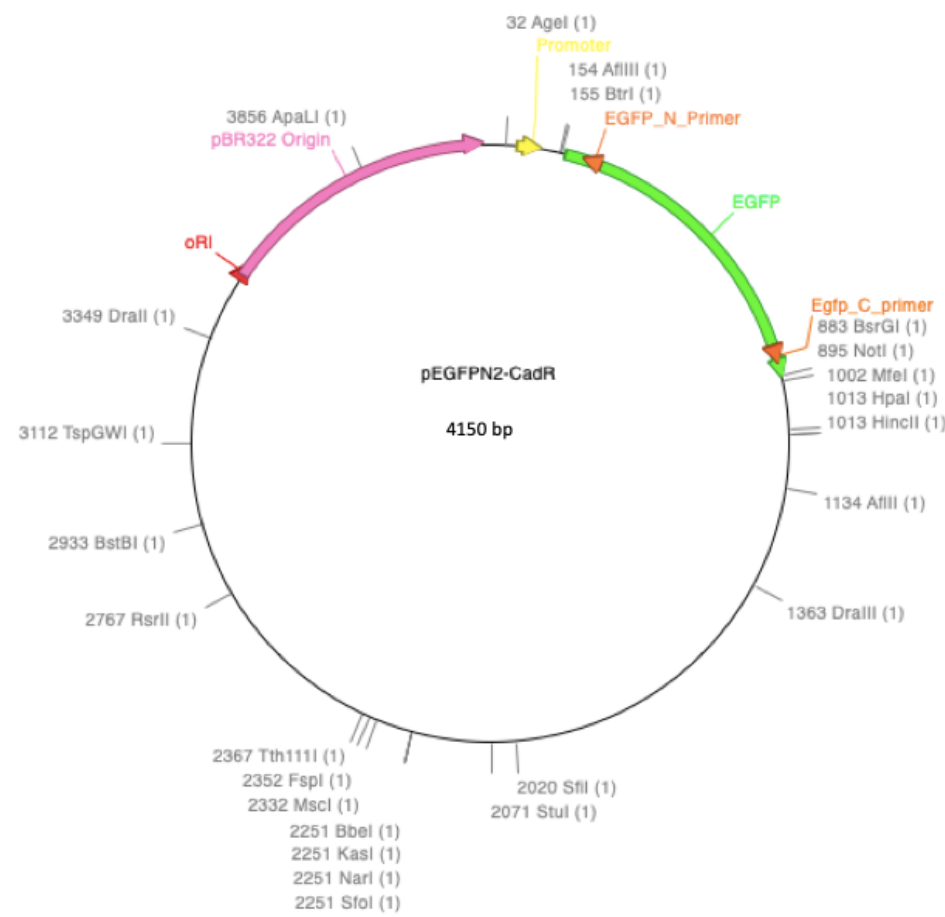

Figure 1. A diagrammatic representation of the vector construct of pEGFP-N2 ${ }_{\text {Cadr }}$ made using Ape software.

2.2. Assessing and measuring GFP expression using metal assays. 
(Table 3) were tested along with negative control to compare the results. The metal concentrations were chosen according to the journal paper by Prabhakaran et al. [8]. Prabhakaran et al., tested their biosensor at $0.5 \mathrm{mM}$ and $1 \mathrm{mM}$. As the incubation periods used for testing their sensor were not mentioned in their research paper, various concentrations had to be tested on this biosensor with different incubation times ( $4 \mathrm{~h}, 8 \mathrm{~h}$, and $24 \mathrm{~h}$ ) to assess the best time frame and metal concentration for the sensor. The biosensor was grown overnight in LB broth supplemented with kanamycin. The cells were grown in varying metal solutions concentrations for 4 hours at $37^{\circ} \mathrm{C}$ with shaking at $200 \mathrm{RPM}$. After pelleting down, the cells were washed and resuspended with $0.85 \% \mathrm{~W} / \mathrm{V} \mathrm{NaCl}$. The liquid was transferred into a 96 well plate forming triplicates to measure the fluorescence intensity (table 4). The cells were also smeared onto a glass slide. They were viewed under a fluorescence microscope to obtain the microscopic image of the fluorescing E. coli. The images were captured with the help of the SIBC Nikon Imaging Center.

\section{Results and Discussion}

\subsection{Construction of the cadmium biosensor.}

The promoter region responsible for driving the GFP gene expression was determined along with the primers mentioned in the journal paper.

>contig25.3 dna:supercontig supercontig:PUPa3_1.0:contig25.3:15957:16231:1
TGCCGATAGTTGCCTTCGCTACGCGCGGGCTCGGGCAACAGGCCTTCGCGCTCGTAGTAGCGGATGGT
CTCCACCGGGCAACCGGTTCTCTTCGCCAGCTCACCGATCTTCATGCCAACCCTCCTCCAATCGCCGACGC
GGCTACCCGAATTGGCAGTACCGCTTGACTCTGTAGTTGCTACAGGGTGTGCAATCGCACCCAACACGT
CAAACGGGAATTGTTTCCATGAGCCACGAACACGCCGACACTTGCTGTCACGGTCACGGACATGATC

Figure 2. Promoter region (highlighted in yellow) and primer sequences (bold and underlined) with a flanking region of 50bp on each side of the promoter. The sequence's total size between forward and reverse primers is $173 \mathrm{bp}$ with the promoter size being $50 \mathrm{bp}$ ).

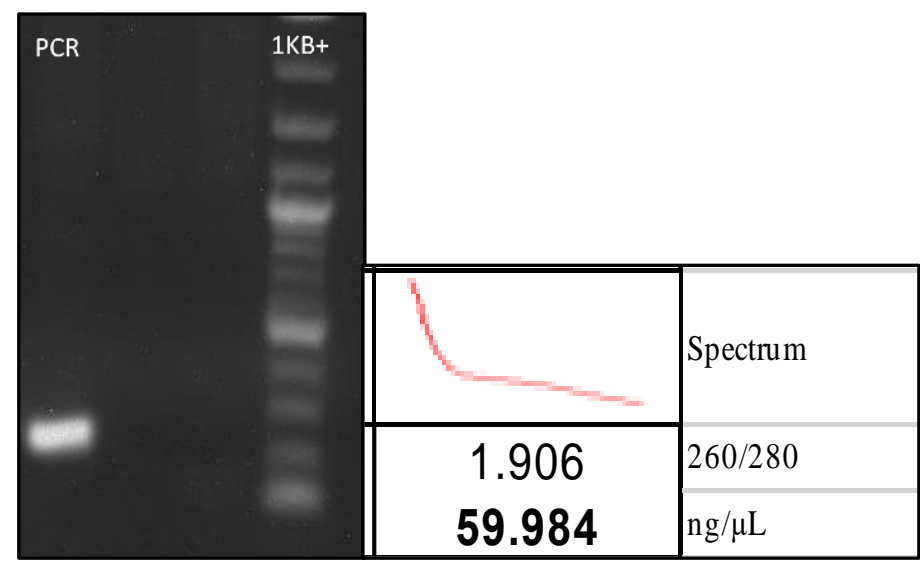

Figure 3. Gel electrophoresis and DNA quantification of the purified product obtained using genomic DNA PCR. The band size of about $200 \mathrm{bp}$ (expected band size $173 \mathrm{bp}$ ) is visible on the left with $1 \mathrm{~kb}+$ ladder on the right. The concentration of the purified product is $59.984 \mathrm{ng} / \mu \mathrm{L}$.

Using the primer pair mentioned in table 2, The promoter region was successfully identified as observed in figure 2 . The product was amplified and purified as observed in figure 3. 
On performing a pairwise alignment using the EMBOSS pairwise alignment needle tool, the obtained and the desired sequences had a similarity percentage of $81.6 \%$. The sequence was also blasted against $P$. aeruginosa genome using NCBI BLAST tool, revealing that the region was a $\mathrm{Cd}(\mathrm{II}) / \mathrm{Pb}(\mathrm{II})$ - responsive transcriptional regulator. After validating the sequencing results, the product was successfully cloned into pEGFP-N2 vector using Gibson assembly resulting in pEGFP-N2CadR. This was then transformed into host competent $E$. coli cells.

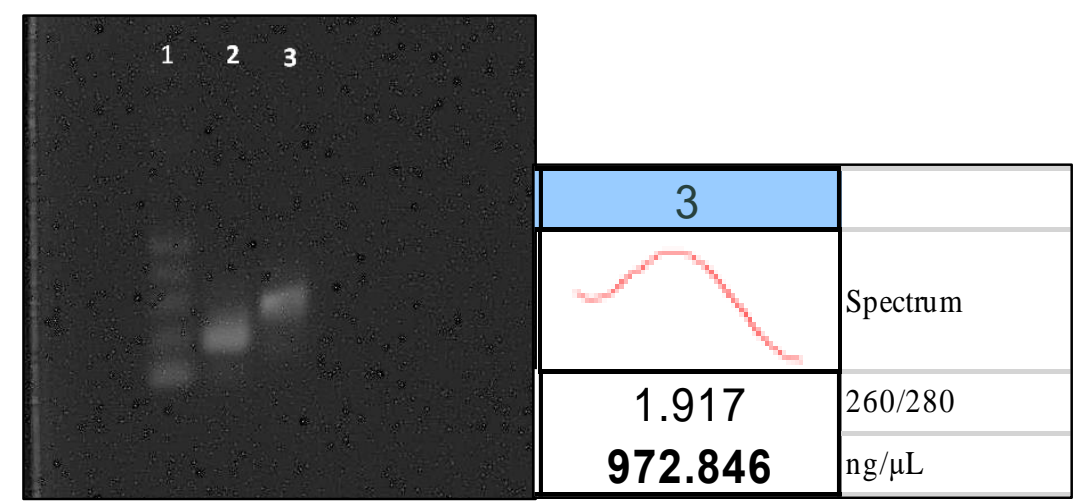

Figure 4. Gel electrophoresis and DNA quantification of the Gibson assembly amplified product obtained in lane 3 . The band size of about $300 \mathrm{bp}$ (expected band size $217 \mathrm{bp}$ ) is visible with $1 \mathrm{~kb}+$ ladder in lane 1 . The concentration of the amplified fragment is $972.846 \mathrm{ng} / \mu \mathrm{L}$.

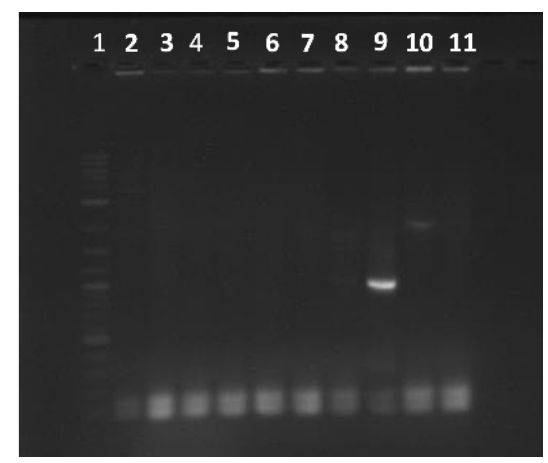

Figure 5. Gel electrophoresis for pEGFP-N2 CadR. An estimated band size of 4150 bp (3973(vector) + 177(insert) is visible in lane 9 with $1 \mathrm{~kb}+$ ladder for comparison in lane 1 .

Figures 4 and 5 show the gel electrophoresis reaction for the Gibson assembly reaction. The amplified promoter region is shown in lane 3 in figure 4. Upon completing the Gibson assembly reaction, the complete product consisting of the vector and the promoter combined was only visible in lane 9 . This signified that the reaction was not entirely successful for all the colonies of bacteria.

The sequencing results of the Gibson assembly clone revealed some more differences in the nucleotide base pairs. Pairwise alignment was performed to compare the base pairs of Prabhakaran's promoter sequence and the promoter sequence after the Gibson assembly reaction. This revealed that the similarity percentage had dropped from $81.6 \%$ to $72.6 \%$.

\subsection{Quantitative analysis of metal assays.}

The sensor was incubated with cadmium chloride, lead nitrate, and copper sulfate at incubation intervals of 4 hours, 8 hours, 12 hours, and 24 hours. The fluorescence was viewed using the SmartView Pro imager system UVCI-1000 series from significant science. The 
biosensor worked best after 4 hours of incubation and responded to higher concentrations of the metal solution when the bacteria were grown to an OD of 0.6 before testing with the metal solutions. When the optical density was not considered, the cell growth was inhibited by the metal solution. Hence, the sensor could not be tested with a higher concentration.

Following this, a wide range of concentrations was tested on the metal ranging from 1 $\mu \mathrm{M}$ to $1 \mathrm{mM}(1000 \mu \mathrm{M})$. The highest concentration tested was $1 \mathrm{mM}$ because it was observed that for the cells not grown to a specific OD, cadmium chloride concentration of more than 2 $\mathrm{mM}$ was toxic to the sensor and fatal if it exceeded $10 \mathrm{mM}$.

The quantitative analysis was performed on the 96 well plates using the Nikon Inverted Microscope Eclipse Ti-E. The images in figure 8 were captured at a magnification of $40 \mathrm{X}$ with CFI60 S Plan Fluor ELWD 40X ADL. The wells' mean intensities were noted using NISElements Advanced Research Software for statistical analysis of the data (figure 6, table 4).

Table 3. Concentrations and different metals solution arrangement triplicates in a 96 well ELISA plate used at the Nikon imaging center.

\begin{tabular}{c|c|c|c|c|c|c|c|c} 
Well A & $0 \mu \mathrm{M}$ & $1 \mu \mathrm{M}$ & $10 \mu \mathrm{M}$ & $50 \mu \mathrm{M}$ & $500 \mu \mathrm{M}$ & $1000 \mu \mathrm{M}$ & $\mathrm{Pb}-50 \mu \mathrm{M}$ & $\mathrm{Cu}-50 \mu \mathrm{M}$ \\
\hline Well B & $0 \mu \mathrm{M}$ & $1 \mu \mathrm{M}$ & $10 \mu \mathrm{M}$ & $50 \mu \mathrm{M}$ & $500 \mu \mathrm{M}$ & $1000 \mu \mathrm{M}$ & Pb-50 $\mu \mathrm{M}$ & $\mathrm{Cu}-50 \mu \mathrm{M}$ \\
\hline Well C & $0 \mu \mathrm{M}$ & $1 \mu \mathrm{M}$ & $10 \mu \mathrm{M}$ & $50 \mu \mathrm{M}$ & $500 \mu \mathrm{M}$ & $1000 \mu \mathrm{M}$ & $\mathrm{Pb}-50 \mu \mathrm{M}$ & $\mathrm{Cu}-50 \mu \mathrm{M}$
\end{tabular}

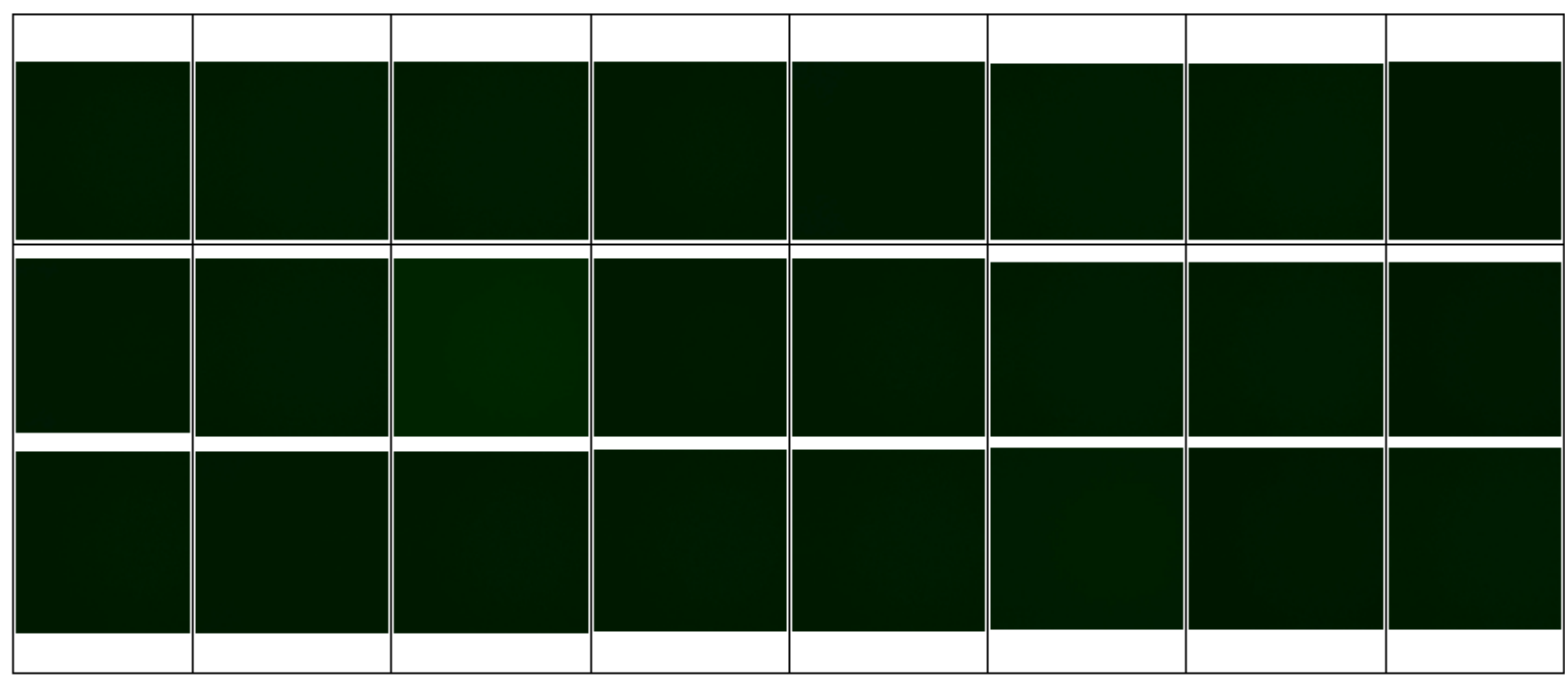

Figure 6. A comparison of the mean fluorescent intensity in wells A, B, and C follows the arrangement in table 2.

Table 4. A comparison of mean fluorescence intensity values at different concentrations of the metal in well A,

\begin{tabular}{c|c|c|c}
\multirow{2}{*}{ Conc $(\mu \mathrm{M})$} & $\mathrm{A}$ & $\mathrm{B}$ & $\mathrm{C}$ \\
\hline 0 & 617.4 & 582.33 & 525.02 \\
\hline 1 & 591.03 & 614.96 & 577.78 \\
\hline 10 & 628.98 & 569.13 & 580.07 \\
\hline 50 & 566.91 & 532.13 & 584.61 \\
\hline 500 & 631.15 & 600.71 & 672.06 \\
\hline 1000 & 604.95 & 614.6 & 605.52 \\
\hline $\mathrm{Pb}-50$ & 433.69 & 474.19 & 474.13
\end{tabular}

As observed in figure 6 , the wells with different metal concentrations had an insignificant difference. In analyzing table 4, it was further clear that the fluorescent intensities at different cadmium concentrations and between lead, cadmium, and copper were not different from each other.

Results for the triplicates were analyzed statistically by plotting a bar chart using Microsoft Excel. The mean and standard deviation were calculated to plot the graph with error 
bars that signified the difference between all the metals' triplicate values at different concentrations (figure 7).

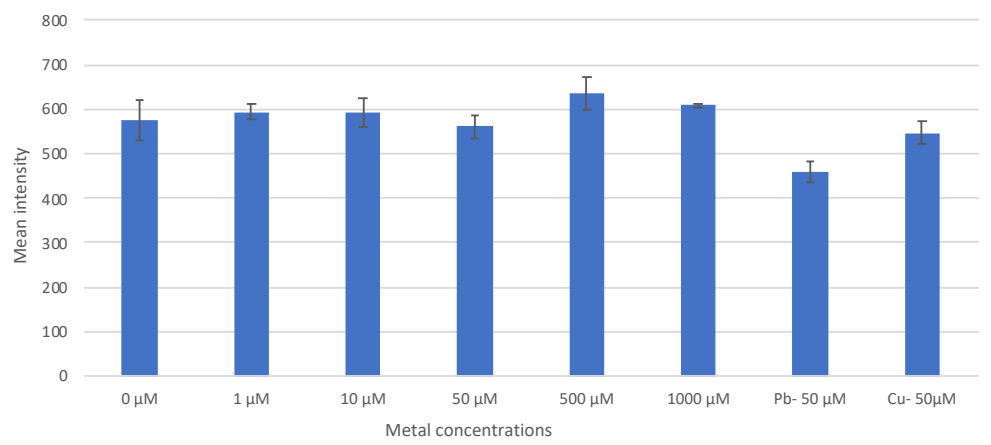

Figure 7. A bar chart comparing of mean fluorescence intensities of triplicates $(n=3)$ at different metal concentrations revealing a non-significant difference within and between all the metals $(\mathrm{p}>0.999$, One-way ANOVA, multiple comparisons performed using Bonferroni's post-hoc test). The error bars represent the standard deviation in the values.

Statistical analyses were performed using the graph-pad prism software. On performing a one-way ANOVA, it was proven that there was no significant difference in the fluorescent intensities between various concentrations of the metals and between different metals.

\subsection{Qualitative analysis of the metal assays.}

The qualitative analysis for fluorescence intensities was performed using Nikon Upright Microscope Eclipse Ni-E. The microscopy slides' images were captured at a magnification of 100X with CFI60 Plan Apochromat VC 100X Oil (figure 8). For the detection of GFP, a GFP-B (GFP bandpass) filter was applied.

\begin{tabular}{|c|c|c|c|c|c|c|c|}
\hline $0 \mu \mathrm{M}$ & $1 \mu \mathrm{M}$ & $10 \mu \mathrm{M}$ & $50 \mu \mathrm{M}$ & $500 \mu \mathrm{M}$ & $1000 \mu \mathrm{M}$ & $\mathrm{Pb}-50 \mu \mathrm{M}$ & $\mathrm{Cu}-50 \mu \mathrm{M}$ \\
\hline & & & & & & & $\cdots$ \\
\hline Well A1 & Well A2 & Well B3 & Well B4 & Well C5 & Well C6 & Well A7 & Well C8 \\
\hline
\end{tabular}

Figure 8. A comparison of the microscopic images of fluorescing $E$. coli taken under a fluorescence microscope. The images reveal a weak fluorescence at all metal concentrations with high background fluorescence and signal interference.

\subsection{Discussion.}

Prabhakaran, et al., 2018 had tested their biosensor with various other metals. It concluded their biosensor responded better to cadmium compared to all other metals tested [8]. In this study, considering that the gene was a $\mathrm{Cd}(\mathrm{II}) / \mathrm{Pb}(\mathrm{II})$ - responsive transcriptional regulator, the biosensor was also tested for its responsiveness against lead. As observed in table 4 , the mean fluorescence intensity in response to $50 \mu \mathrm{M}$ of lead nitrate is lower than cadmium. The biosensor was also checked for responsiveness towards copper using $50 \mu \mathrm{M}$ copper sulfate solution. On comparing the results, the response to copper was similar to cadmium's response; however, the intensity was slightly lower than the intensity in response to cadmium, as observed by the graph trends. It was concluded that the biosensor's response to cadmium was 
statistically equal to its response to lead and copper. According to table 4, the sensor's best response to the metal cadmium was at $500 \mu \mathrm{M}$ or $0.5 \mathrm{mM}$ and $1000 \mu \mathrm{M}$ or $1 \mathrm{mM}$, which is also consistent with Prabhakaran and his colleagues' results [8]. The intensity values of the negative control and other metals were similar. This might be due to the leaky expression of the cells. However, on observing the image in figure 8, the negative control shows a dim expression compared to other concentrations. The lead image also shows a dimmer expression; however, the bacteria seem to respond better to copper. The results obtained were vague and inconclusive. This might have occurred due to an increased number of mutations in the sequences.

On comparing the intensities at increasing concentrations of the metal, no significant difference was observed. There was no increasing intensity gradient observed with an increase in the cadmium chloride concentration. This could also be attributable to the mutations in the sequence.

On comparing the sequence obtained from the journal paper with the sequence obtained after Gibson assembly cloning, there was a difference of $9.4 \%$ in their base pairs. Following Gibson assembly, another pairwise alignment was conducted between the Gibson assembly clone and PA7 intergenic region. An identity of $76 \%$ was recorded. Following cloning, plasmid extraction, and sequencing, the sequence of the clone had mutated significantly. This might have reduced the strength of the promoter. It is also possible that the strain (BC15) used by Prabhakaran and his colleagues had a stronger expression to cadmium and did not respond to other metals due to it being isolated from industry effluent water waste [8]. This might have resulted in a higher resistance and a better response to cadmium. Whereas, in this study, the strain of bacteria used was different and was not isolated from an environment abundant in cadmium leading to a weaker expression of GFP due to the promoter not being comparatively strong.

The sensitivity, specificity, and the detection properties of the whole-cell biosensor are dependent on the reporter genes it uses [9]. Apart from GFP, other reporter genes that can be incorporated successfully into the biosensor include the luc (firefly luciferase), lux (bacterial luciferase), and LacZ (beta galactosidase). GFP holds an advantage over the other reporter genes as it does not require a substrate and is highly stable; however, some of the disadvantages of GFP include low sensitivity, lag-time for stable fluorescence, and autofluorescence [9]. These disadvantages might also have affected this study as the reporter gene's intrinsic fluorescence might have caused increased background fluorescence and signal interference leading to decreased sensitivity of the biosensor. In comparison, the sensors based on lux and lacZ can detect the metal with more sensitivity [9].

However, in a study by Joe et al., 2012, the researchers developed a cadmium biosensor from Deinococcus radiodurans. They used lacZ as the reporter gene [13]. The detection limit of their cadmium biosensor was 1-10 mM. The biosensor developed in this study can detect cadmium concentrations as low as $1 \mu \mathrm{M}$ and possibly lower, making this a better biosensor than the one developed by Joe et al. [13].

\section{Conclusions}

In conclusion, the aim of this experiment of constructing a cadmium biosensor was partly achieved. The sensor was not classified as completely specific to cadmium. Although it could detect low concentrations of cadmium, the intensity was not very high. However, due to time constraints, further tests could not be performed on the sensor. Being a promoter from a 
different strain, it is possible that the biosensor has a higher affinity towards different concentrations of the metal or responds better to a completely different metal not tested in this study as the promoter is a part of the MerR family of regulatory proteins that respond well to other metals as well. As Prabhakaran, et al., the response to the metal depends on the accessibility of the metal ions to the promoter region [8]. Hence, further studies need to be performed to determine the biosensor's sensitivity and specificity towards different metals at different concentrations.

The biosensor should be tested with actual cadmium contaminated water samples to test for the efficacy of its sensing ability. To solve the problem of leaky expression, the vector can be transformed into E. coli BL21(DE3), which has proven to aid this problem [14]. In silico studies can be performed to analyze the exact promoter region of the bacteria. These studies can reveal the binding affinities and interactions of other genes with the promoter region. As shown in some studies, the bend of the MerR family promoters results in the shortening of the spacer between -35 and -10 promoter elements causing a threefold increase in the binding affinity [15]. Using 3D model software, the promoter region's specificity and sensitivity can be increased using site-directed mutagenesis [15]. As proposed by Lee, et al., the biosensor's eGFP region can be modified by inserting metal-binding loops between $\beta$-strands 9 and 10 of the eGFP, making the sensor completely specific to a particular metal, which in this case is cadmium [15]. The microbial sensors can be combined with micro/nanotechnology for easier detection. The system should also be made portable to make it convenient for practical applications. Moreover, the integration of micro- or nano-systems in the biosensors can result in greater sensitivity and specificity, allowing an accurate and rapid response time [16].

\section{Funding}

I am thankful to the Management and Development School of Singapore for funding my undergraduate final year project as a part of the course requirements.

\section{Acknowledgments}

I would like to express my sincere gratitude to my supervisor Dr. Poon Kar Lai, for her constant support and able guidance throughout the completion of my project. I would especially like to thank my group members Aw Zheng Hao, Wong Siu Ling, and Tong Bao Zhu, who always provided motivation and help despite working on separate projects. Lastly, my sincere thanks also go to the lab technicians Ms. Sinthu and Ms. Dhivegaa, for their technical support.

\section{Conflicts of Interest}

The authors declare no conflict of interest.

\section{References}

1. Monisha, J.; Tenzin, T.; Naresh, A.; Blessy, B.M.; Krishnamurthy, N.B. Toxicity, mechanism and health effects of some heavy metals. Interdisciplinary Toxicology 2014, 7, 60-72, https://doi.org/10.2478/intox2014-0009.

2. RoyChowdhury, A.; Datta, R.; Sarkar, D. Chapter 3.10-Heavy Metal Pollution and Remediation. In: Green Chemistry. Török, B.; Dransfield, T. Eds. Elsevier: 2018; pp. 359-373, https://doi.org/10.1016/B978-0-12809270-5.00015-7.

3. Gutiérrez, J.C.; Amaro, F.; Martín-González, A. Heavy metal whole-cell biosensors using eukaryotic microorganisms: an updated critical review. 2015, 6, 1-48, https://doi.org/10.3389/fmicb.2015.00048. 
4. Marzan, L.W.; Hossain, M.; Mina, S.A.; Akter, Y.; Chowdhury, A.M.M.A. Isolation and biochemical characterization of heavy-metal resistant bacteria from tannery effluent in Chittagong city, Bangladesh: Bioremediation viewpoint. The Egyptian Journal of Aquatic Research 2017, 43, 65-74, https://doi.org/10.1016/j.ejar.2016.11.002.

5. Rodrigue, J.; Champoux, L.; Leclair, D.; Duchesne, J.-F. Cadmium concentrations in tissues of willow ptarmigan (Lagopus lagopus) and rock ptarmigan (Lagopus muta) in Nunavik, Northern Québec. Environmental Pollution 2007, 147, 642-647, https://doi.org/10.1016/j.envpol.2006.10.017.

6. Rafati-Rahimzadeh, M.; Rafati-Rahimzadeh, M.; Kazemi, S.; Moghadamnia, A. Cadmium toxicity and treatment: An update. babol-caspjim 2017, 8, 135-145, https://doi.org/10.22088/cjim.8.3.135.

7. Capitelli, F.; Colao, F.; Provenzano, M.R.; Fantoni, R.; Brunetti, G.; Senesi, N. Determination of heavy metals in soils by Laser Induced Breakdown Spectroscopy. Geoderma 2002, 106, 45-62, https://doi.org/10.1016/S0016-7061(01)00115-X.

8. Prabhakaran, R.; Rajkumar, S.N.; Ramprasath, T.; Selvam, G.S. Identification of promoter PcadR, in silico characterization of cadmium resistant gene cadR and molecular cloning of promoter PcadR from Pseudomonas aeruginosa BC15. Toxicology and Industrial Health 2018, 34, 819-833, https://doi.org/10.1177/0748233718795934.

9. Gui, Q.; Lawson, T.; Shan, S.; Yan, L.; Liu, Y. The Application of Whole Cell-Based Biosensors for Use in Environmental Analysis and in Medical Diagnostics. Sensors 2017, 17, https://doi.org/10.3390/s17071623.

10. Donaldson, T.; Dattelbaum, J. Development of whole cell biosensors mediated by bacteria chemoreceptors (614.5). The FASEB Journal 2014, 28, 50-65, https://doi.org/10.1096/fasebj.28.1_supplement.614.5.

11. Yagi, K. Applications of whole-cell bacterial sensors in biotechnology and environmental science. Applied Microbiology and Biotechnology 2007, 73, 1251-1258, https://doi.org/10.1007/s00253-006-0718-6.

12. SGI-DNA,. Gibson assembly cloning guide, San Diego : SGI-DNA, Inc. 2019. Assessed on 6 Aug 2019. Available from: http://blog.sgidna.com/2016/08/how-does-gibson-assembly-cloning.html

13. Joe, M.-H.; Lee, K.-H.; Lim, S.-Y.; Im, S.-H.; Song, H.-P.; Lee, I.S.; Kim, D.-H. Pigment-based whole-cell biosensor system for cadmium detection using genetically engineered Deinococcus radiodurans. Bioprocess and Biosystems Engineering 2012, 35, 265-272, https://doi.org/10.1007/s00449-011-0610-3.

14. Briand, L.; Marcion, G.; Kriznik, A.; Heydel, J.M.; Artur, Y.; Garrido, C.; Seigneuric, R.; Neiers, F. A selfinducible heterologous protein expression system in Escherichia coli. Scientific Reports 2016, 6, https://doi.org/10.1038/srep33037.

15. Lee, W.; Kim, H.; Kang, Y.; Lee, Y.; Yoon, Y. A Biosensor Platform for Metal Detection Based on Enhanced Green Fluorescent Protein. Sensors 2019, 19, https://doi.org/10.3390/s19081846.

16. Lim, J.W.; Ha, D.; Lee, J.; Lee, S.K.; Kim, T. Review of Micro/Nanotechnologies for Microbial Biosensors. 2015, 3, 61-65, https://doi.org/10.3389/fbioe.2015.00061. 